\title{
Otimização reológica de suspensões aquosas de óxido de ferro (III)
}

Renata Fumagali Scirea ${ }^{1}$, Luiz Eloi Vieira Junior ${ }^{1}$ João Batista Rodrigues Neto ${ }^{1}$, Dachamir Hotza ${ }^{2}$

\author{
${ }^{1}$ Laboratório Interdisciplinar de Materiais (LABMAT) \\ Departamento de Engenharia Mecânica (EMC) \\ Universidade Federal de Santa Catarina (UFSC) \\ 88040-900, Florianópolis, SC \\ e-mail: renata_scirea@hotmail.com, eloi_junior@hotmail.com,jbrn.ufsc@gmail.com \\ ${ }^{2}$ Laboratório de Materiais Cerâmicos e Compósitos (CERMAT) \\ Departamento de Engenharia Química (EQA) \\ Universidade Federal de Santa Catarina (UFSC) \\ 88040-900, Florianópolis, SC \\ e-mail: d.hotza@ufsc,br
}

\begin{abstract}
In this work the dispersion of iron oxide III particles in aqueous media was studied. To achieve the maximum solid content zeta potential measurements were carried as a function of $\mathrm{pH}$. The influences of two different types of polyacrylates as dispersants were investigated. Suspensions were produced by varying solids concentration up to $30 \mathrm{vol} \%$. The results showed a strong influence of $\mathrm{pH}$ on the size distribution of the particles in suspension. The most efficient dispersant towards particle dispersion was sodium polyacrylate. The maximum solid content in the suspension was optimized to 25 vol \% with density values higher than $35 \%$ theoretical density with $2 \mathrm{wt}$ \% polyacrylate.
\end{abstract}

Keywords: Colloidal processing, rheology, iron oxide III.

\section{INTRODUÇÃO}

O óxido de ferro é abundante na natureza e utilizado num vasto campo tecnológico. Há três formas de óxidos de ferro: óxido de ferro (II), $\mathrm{FeO}$ (wustita); óxido de ferro (II,III), $\mathrm{Fe}_{3} \mathrm{O}_{4}$ (magnetita); óxido de ferro (III), $\mathrm{Fe}_{2} \mathrm{O}_{3}$ (fases $\alpha, \beta, \gamma$, ou $\varepsilon$ ). $0 \mathrm{~A}$ hematita $\left(\mathrm{Fe}_{2} \mathrm{O}_{3}\right.$, fase $\alpha$ ) é usada principalmente como pigmento para tintas $\mathrm{e}$ vidros [ $\underline{2}-\underline{6}$ ], tratamento de água e sensores eletroquímicos. [ $\underline{7}-\underline{9}$ ] Quando dopados com metais nobres, como por exemplo, platina e ouro, pode ser aplicado na produção de hidrogênio e na fabricação de anodos0pelas suas propriedades de estabilidade térmica e estrutural. Outras formas de óxido de ferro como a magnetita $\left(\mathrm{Fe}_{3} \mathrm{O}_{4}\right)$ e a maghemita $\left(\mathrm{Fe}_{2} \mathrm{O}_{3}\right.$, fase $\left.\gamma\right)$, possuem características importantes como biomateriais magnéticos (ferrites) aplicados na medicina e farmácia. Na medicina, a magnetita é usada como forma de transporte de fármacos e contrastes em ressonância. 0

Conceitualmente, suspensões são misturas heterogêneas constituídas de sólidos particulados que estão dispersas em um meio líquido. Durante a sua etapa de produção, é necessário que as partículas permaneçam individualizadas no meio líquido. Isto acarreta em uma série de benefícios de processamento, como a elevada concentração de sólidos, gerando peças com menos retração durante a secagem, consequentemente, após a sinterização será alcançada uma microestrutura mais homogênea e um componente com melhores propriedades mecânicas. São ditas estabilizadas quando o balanço das forças repulsivas supera as atrativas. Isso pode ser alcançado de três formas: (i) repulsão eletrostática, que consiste na manutenção das partículas em suspensão num meio líquido polar (água ou solvente orgânico) através do desenvolvimento de uma nuvem iônica ou dupla camada elétrica. Desta forma, ao se inserir pós em um meio líquido, instantaneamente se forma uma nuvem de cargas elétricas ao seu redor, conhecida como dupla camada elétrica. Esta região é formada por uma primeira camada de íons de sinais opostos (contra-íons) que está firmemente adsorvida a superfície por atração eletrostática e uma segunda camada mais externa (camada difusa). A região de interface entre as duas camadas é chamada camada de Stern e é nesta região onde ocorre o cisalhamento durante o fluxo de líquido. 
A diferença de potencial entre a camada difusa e a dispersão eletricamente neutra (potencial zeta, $\zeta$ ) pode ser determinada experimentalmente. $O$ potencial zeta é um parâmetro importante para a avaliação da estabilidade de uma suspensão; (ii) impedimento estérico que se baseia na adsorção à superfície de moléculas orgânicas, geralmente poliméricas, que atuam como agentes de repulsão estérica. Para o polímero agir efetivamente, deve haver interação entre a superfície da partícula e o polímero utilizado, caso contrário pode ocorrer o efeito contrário indesejado à adsorção, que é a dessorção. Além disso, a espessura da camada de recobrimento deve ser extensa o suficiente para não entrar na distância de forte atuação das forças de van der Waals. Por último, o polímero ainda precisa apresentar afinidade entre o líquido. Se comparada com o mecanismo eletrostático, o mecanismo estérico apresenta vantagens como pouca sensibilidade aos eletrólitos, permite a variação do meio líquido, possibilita a produção de suspensões com maior fração de sólidos, exibe reversibilidade à floculação e; (iii) eletrostérico, na qual são utilizados polímeros específicos com grupos ionizáveis que adsorvem às partículas, também conhecidos como polieletrólitos. Outros compostos como fosfatos, citratos e sulfatos também promovem o mesmo efeito. No mecanismo eletrostérico, a carga superficial da partícula passa a ser influenciada também pela carga elétrica desenvolvida pela dissociação do eletrólito. [12-16] A partir de uma suspensão estável, diversas são as técnicas de processamento para se obter geometrias diferentes e densificação elevada, com acabamentos superficiais e tolerâncias dimensionais adequados. Para isso é necessário conhecer o comportamento reológico das suspensões, particularmente as que usam base aquosa. $\mathrm{O}$ objetivo deste trabalho é estudar a dispersão de partículas submicrométricas de hematita em meio aquoso a partir do conhecimento das características de cargas superficiais e tipos de dispersantes para se maximizar a concentração de sólidos sem comprometer as características essenciais desejadas de um componente sinterizado denso.

\section{MATERIAIS E MÉTODOS}

Hematita usada como pigmento vermelho (Colorminas, Brasil) foi o material de partida. O tamanho de partícula e o potencial zeta em função do $\mathrm{pH}$ foram medidos no mesmo equipamento (Zetasizer-Nanosizer Zen 3600 , Malvern). Às suspensões foram adicionados $\mathrm{HCl}(1 \mathrm{M})$ e $\mathrm{NaOH}(0,25 \mathrm{M})$ para efetuar as medições na faixa de $\mathrm{pH}$ de 2 a 12. A estabilização das suspensões será de acordo com o mecanismo eletrostérico, devido a sua ampla faixa de atuação. Foram estudados dois tipos de dispersantes comerciais: poliacrilato de sódio, Liosperse ${ }^{\circledR} 510$ e de amônio, Liosperse ${ }^{\circledR} 511$ (PANa e PAA, Miracema- Nuodex, Brasil). A concentração volumétrica inicial de sólidos foi fixada em $10 \%$. Inicialmente, foram adicionadas às suspensões $1 \% \mathrm{em}$ massa de cada tipo de dispersante em relação à quantidade de sólidos. A seguir, foram realizadas medições com adições crescentes de dispersante até $3,0 \% \mathrm{~m} / \mathrm{m}$. O comportamento reológico foi medido por meio de um viscosímetro rotacional com geometria de cilindros concêntricos (VT550, Haake, Alemanha) a uma taxa de cisalhamento de 1 a $3000 \mathrm{~s}^{-1} 3 \mathrm{~min}$, mantendo-se a $3000 \mathrm{~s}^{-1}$ durante 1 min e volta a $0 \mathrm{em} 3 \mathrm{~min}$. O ajuste das curvas reológicas foi feito a partir do modelo de Casson:

$$
\tau^{0,5}=\tau_{0}^{0,5}+\left(\eta_{c} \cdot \dot{\gamma}\right)^{0,5}
$$

onde: $\tau_{0}$ é a tensão cisalhante de escoamento e $\eta$ a viscosidade ajustados ao modelo de Casson.

O livre caminho médio, $\lambda$, foi calculado obedecendo ao modelo de Fullmann 0 :

$$
\lambda=\frac{\frac{2}{3} \cdot d \cdot(1-\Phi)}{\Phi}
$$

onde: d é o diâmetro das partículas e $\phi$ a concentração volumétrica de sólidos na suspensão.

A colagem se deu num molde de silicone suportado numa placa de gesso. Após o vazamento das suspensões, as amostras foram removidas do molde e deixadas ao ar para a secagem por $24 \mathrm{~h}$. As densidades foram medidas pelo método de Arquimedes em água nas amostras verdes e sinterizadas (média de três medidas). A microscopia eletrônica de varredura (Phillips XL30, Holanda) foi utilizada para analisar a morfologia das partículas e a superfície de fratura das amostras coladas.

\section{RESULTADOS E DISCUSSÕES}

\subsection{Tamanho de partícula e potencial zeta}

O tamanho de partícula é fortemente influenciado pelo pH. Os resultados obtidos são mostrados na Figura 1. A curva de potencial zeta apresenta um potencial negativo numa extensa faixa de $\mathrm{pH}$, indicando que a super- 
fície das partículas exibe cargas predominantemente negativas com o ponto de carga zero situado em $\mathrm{pH}$ aproximadamente 6 . Na literatura são encontrados valores de pontos isoelétricos para a hematita variando desde 4,2 até $9,4[\underline{4}-\underline{6}, \underline{11}, \underline{18}, \underline{19}]$. Isso se deve a quantidade de impurezas presentes na matéria-prima bem como a ativação intencional da superfície para uma aplicação específica. Sendo assim, é plausível afirmar que o valor encontrado está de acordo com a literatura. O potencial zeta alcança um valor máximo em módulo no pH 10 e então aumenta novamente em virtude da saturação de carga elétrica ao redor das partículas resultando em um estados de compressão da dupla camada elétrica. Na região próxima ao PCZ, há uma neutralização das cargas elétricas, o que favorece a aglomeração das partículas. Afastando-se dessa região, há uma melhor dispersão das partículas devido ao aumento da concentração de íons ao redor das partículas. Os resultados experimentais mostraram que o tamanho médio das partículas de hematita variou de 0,6 a $0,9 \mu \mathrm{m}$.

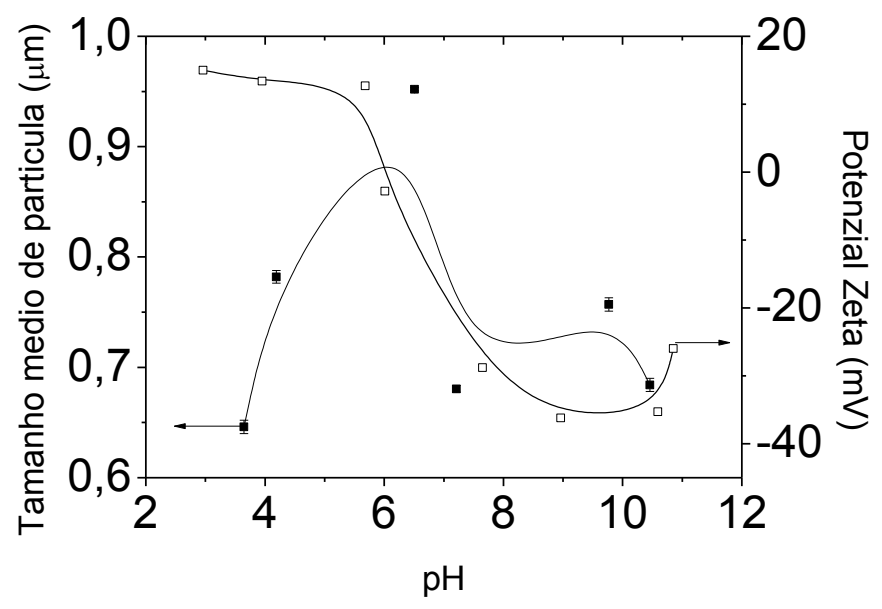

Figura 1: Variação do tamanho de partícula e potencial zeta em função do pH.

\subsection{Tipo de dispersante}

A Figura 2 mostra o comportamento reológico de suspensões de hematita com/sem defloculante. As curvas possuem comportamentos pseudoplásticos e o dispersante mais eficiente na diminuição da viscosidade foi o poliacrilato de sódio. Este resultado está de acordo com resultados já publicados na literatura. 0 Um dispersante efetivo deve possuir valores baixos de valência, pois produzem um decaimento suave na curva de potencial elétrico em função da distância de separação entre partículas. 0

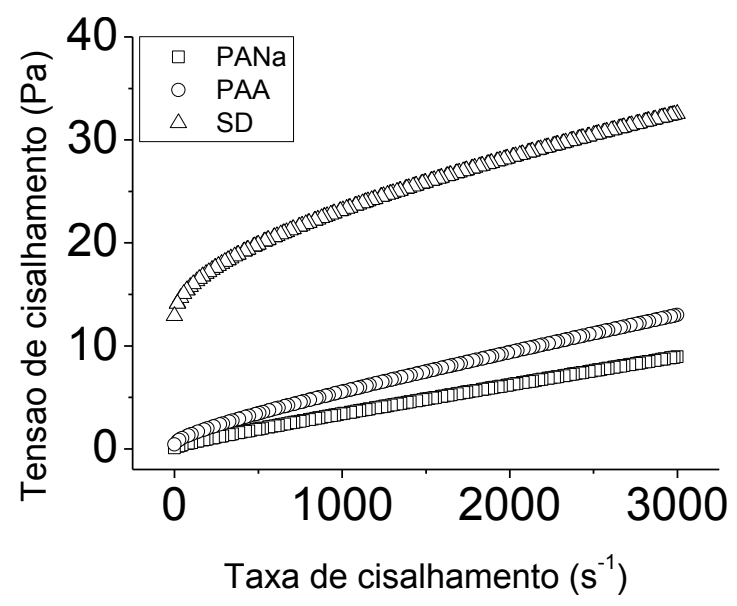

Figura 2: Curvas de fluxo para suspensões aquosas de hematita $(10 \% \mathrm{v} / \mathrm{v})$ sem defloculante (SD) ou com defloculante: poliacrilato de sódio (PANa) e poliacrilato de amônio (PAA) com pH ajustado para 10.

Além dos valores de valência, a condição para o desenvolvimento de uma camada suficientemente extensa para inibir a atuação das forças de van der Waals é o tamanho dos íons. Apesar de ambos os íons sódio e amônio possuírem a mesma carga, os valores dos raios atômicos são distintos: $\mathrm{Na}^{+1}=0,98 \AA$ e $\mathrm{NH}_{4}^{+1}$ $=1,43 \AA$, diferença entre tamanhos de aproximadamente $46 \%$. Poderia se supor que o poliacrilato de amônio 
alcançaria melhor desempenho de dispersão e resultado foi exatamente o contrário. O processo de formação da dupla camada elétrica baseia-se no envolvimento dos íons que estão presentes no líquido que formam ligações com moléculas de água. Estes são chamados de íons hidratados (íons solvatados). Os valores de raios iônicos hidratados são mostrados na Tabela 1. Quando analisados isoladamente, o íon amônio se mostrou maior. Contudo, ao se ligar com a água, o íon hidratado de sódio se mostrou quase $70 \%$ maior que o de amônio o que justifica a maior eficiência do poliacrilato de sódio. 0

Tabela 1: Raios iônicos para cada tipo de cátion. [20 - 22]

\begin{tabular}{c|c|c}
\hline Cátion & $\begin{array}{c}\text { Raio } \\
\text { iônico (̊) }\end{array}$ & $\begin{array}{c}\text { Raio } \\
\text { hidratado (̊) }\end{array}$ \\
\hline $\mathrm{Li}^{+1}$ & 0,78 & 7,3 \\
\hline $\mathrm{Na}^{+1}$ & 0,98 & 5,6 \\
\hline $\mathrm{K}^{+1}$ & 1,33 & 3,8 \\
\hline $\mathrm{NH}_{4}^{+1}$ & 1,43 & 3,3 \\
\hline $\mathrm{Mg}^{+2}$ & 0,78 & 10,8 \\
\hline $\mathrm{Ca}^{+2}$ & 1,06 & 9,6 \\
\hline $\mathrm{Ba}^{+2}$ & 1,43 & 8,8 \\
\hline $\mathrm{Al}^{+3}$ & 0,57 & 4,8 \\
\hline
\end{tabular}

Ensaios preliminares revelaram que a adição de $0,5 \% \mathrm{~m} / \mathrm{m}$ de PANa a curva apresentou pouca alteração do seu comportamento sem a adição do poliacrilato, ou seja, a concentração de dispersante não foi suficientemente para recobrir partículas da suspensão. Contudo, a partir de $1 \% \mathrm{~m} / \mathrm{m}$ percebe-se com maior evidência a melhora no comportamento de fluidez. Na Figura 3, vê-se que a partir de $1 \% \mathrm{~m} / \mathrm{m}$ de PANa, a viscosidade das suspensões foi reduzida de maneira perceptiva, até atingir a concentração de $3 \%$ em massa, quando se alcançou a saturação de polímero na suspensão (sobredefloculação). A explicação típica para o aumento da viscosidade em função da adição de polímeros é o efeito de ponteamento (bridging). Nesse caso, as camadas de polímeros deixam de envolver partículas individuais e aprisionam mais de uma partícula, formando estruturas tridimensionais, elevando sua viscosidade. [17] Com base nos dados apresentados na Figura 4, escolheu-se a composição de $2,0 \% \mathrm{~m} / \mathrm{m}$ de PANa para a confecção das suspensões para posterior colagem, já que com a adição de $2,5 \% \mathrm{~m} / \mathrm{m}$ não houve redução significativa e o ponto de saturação se deu em $3,0 \% \mathrm{~m} / \mathrm{m}$. Por outro lado, a presença em demasia de aditivos na composição da suspensão dificulta a sua eliminação durante a sinterização, aumentando o tempo de processo e por consequência, seus custos de produção.

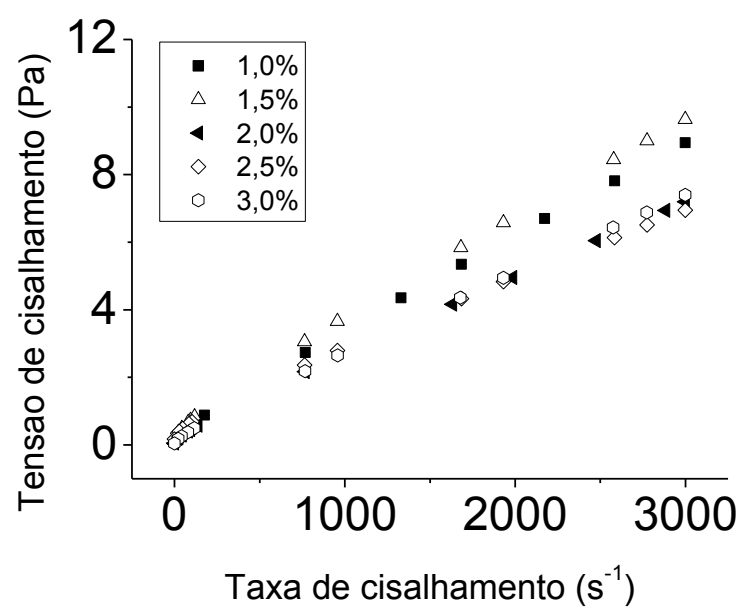

Figura 3: Curvas de fluxo das suspensões aquosas com pH ajustado em 10 variando a concentração de dispersante. 
A Tabela 2 apresenta dados quantitativos a respeito da influência da concentração de PANa nos parâmetros reológicos obtidos a partir do modelo de Casson (Equação 1) e na densificação dos compactados colados a verde. Nota-se que na concentração de $2,0 \% \mathrm{~m} / \mathrm{m}$ de PANa, os valores de tensão inicial de escoamento $\left(\tau_{0}\right)$, a tixotropia da suspensão e sua viscosidade a uma taxa cisalhante baixa são limites e, a partir desta, os valores são aumentados. Outra justificativa para a escolha da concentração de 2,0\% de dispersante é a densificação alcançada dos compactados colados. Concentrações inferiores e superiores a esse valor limite apresentaram dispersões maiores nos valores de densidades a verde. Concentrações baixas não produziram efeito de envolvimento completo e individualização das partículas de hematita, restando ainda aglomerados que são difíceis de acomodar durante a drenagem do líquido na colagem.

Tabela 2: Efeito da concentração de PANa na tensão de inicial de escoamento, tixotropia, viscosidade (a 130 s-1) e densidade a verde.

\begin{tabular}{c|c|c|c|c}
\hline $\begin{array}{c}\text { PANa } \\
(\boldsymbol{\%} \text { massa })\end{array}$ & $\begin{array}{c}\text { Tensão de } \\
\text { escoamento }(\mathbf{P a})\end{array}$ & $\begin{array}{c}\text { Tixotropia } \\
(\mathbf{P a} / \mathbf{s})\end{array}$ & $\begin{array}{c}\text { Viscosidade } \\
(\mathbf{m P a} . \mathbf{s})\end{array}$ & $\begin{array}{c}\text { Densidade a verde } \\
(\boldsymbol{\%} \mathbf{D . T} .)\end{array}$ \\
\hline 0 & 14,14 & 4073 & 123 & $33,19 \pm 4,08$ \\
\hline 0,50 & 13,44 & 3508 & 120 & $31,18 \pm 1,66$ \\
\hline 1,00 & 0,25 & 2118 & 6,00 & $35,30 \pm 0,94$ \\
\hline 1,50 & 0,35 & 1843 & 7,00 & $35,76 \pm 0,62$ \\
\hline $\mathbf{2 , 0 0}$ & $\mathbf{0 , 1 9}$ & $\mathbf{1 1 5 8}$ & $\mathbf{4 , 0 0}$ & $\mathbf{3 7 , 0 5} \pm \mathbf{0 , 3 4}$ \\
\hline 2,50 & 0,35 & 1165 & 6,00 & $37,02 \pm 1,62$ \\
\hline 3,00 & 0,17 & 1149 & 4,00 & $37,07 \pm 2,48$ \\
\hline
\end{tabular}

\subsection{Concentração máxima de sólidos}

Escolhido o tipo de dispersante e sua concentração, investigou-se a concentração máxima de sólidos, ou seja, a máxima concentração de partículas dispersas sem que comprometa a fluidez necessária ao processamento por colagem de barbotina. A Figura 4 mostra os resultados obtidos. As viscosidades das suspensões aumentam significativamente a partir de $20 \%$ v/v., tendo seu máximo em $30 \%$ v/v quando se obteve uma suspensão já com consistência de pasta.

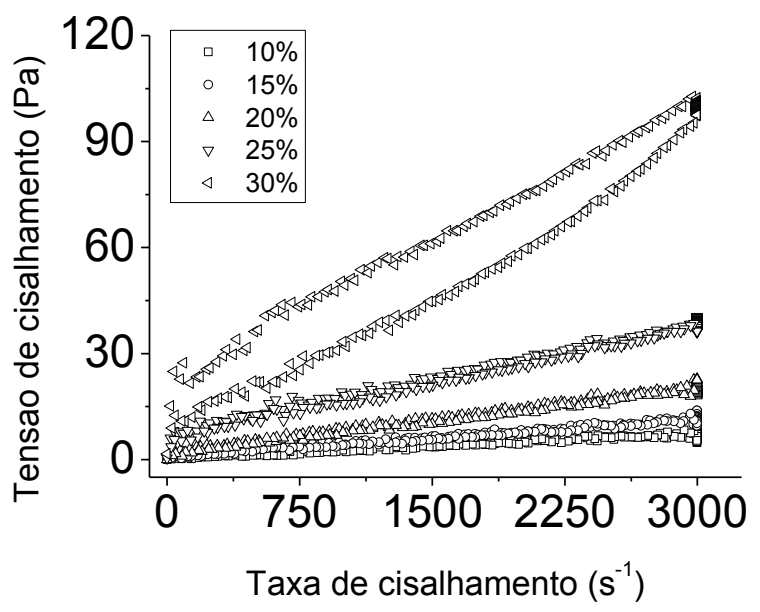

Figura 4: Curvas de fluxo para diferentes concentrações mássicas de sólidos em suspensões de hematita com $2 \%$ de PANa e pH 10.

A Tabela 3 estabelece um comparativo entre variação da concentração de sólidos das suspensões e as suas propriedades reológicas. Nota-se que até $20 \% \mathrm{v} / \mathrm{v}$ de sólidos as características descritas na tabela aumentam com comportamento quase linear. A partir de $25 \% \mathrm{v} / \mathrm{v}$ há um crescimento exponencial desses valores. Isso se deve ao fato de que a partir dessa concentração de sólidos há uma transição no comportamento reológico de suspensão para o de pasta, evidenciados pela elevação nos valores de tixotropia e diminuição do comprimento do livre caminho médio, que fornece um valor de distanciamento entre partículas. As sus- 
pensões com concentrações de sólidos de 10 e $15 \%$ v/v. apresentaram valores menores, ou seja, as partículas de hematita possuíam uma quantidade de líquido suficientemente grande para mantê-las bem dispersas. Por outro lado, ao subir a concentração de sólidos este distanciamento foi diminuindo até se formar um filme de líquido estreito na qual possibilitou o maior número de colisões entre partículas, aumentando a viscosidade das suspensões em consequência da energia liberada a partir destas colisões. Vale ressaltar que a taxa de cisalhamento de 130 s-1 corresponde ao um valor típico na etapa de colagem de barbotina. A decisão na escolha da concentração de sólidos de $25 \%$ v/v. é reforçada analisando os valores das densificações dos compactados colados a verde. Diminuindo o livre caminho médio entre as partículas se torna difícil à formação de canais de drenagem do líquido durante a colagem afetando a sua conformação, produzindo corpos a verde muito irregulares e com menor densidade a verde. A superfície de fratura do compactado colado a verde de hematita é mostrada na Figura 5. Sua morfologia se assemelha a de finos bastonetes com diâmetros e comprimentos médios da ordem de 0,20 e 0,60 $\mu \mathrm{m}$ respectivamente. Estas características comprometem o empacotamento tanto durante o cisalhamento quanto na colagem, daí a rápida transição entre as condições de fluidez das suspensões, necessitando também adicionar grande quantidade de dispersante.

Tabela 3: Efeito da concentração de sólidos na tensão inicial de escoamento, tixotropia, viscosidade (a $130 \mathrm{~s}^{-1}$ ) e livre caminho médio.

\begin{tabular}{c|c|c|c|c|c}
\hline $\boldsymbol{\Phi}(\boldsymbol{\%}$ vol.) & $\boldsymbol{\tau}_{\mathbf{0}}(\mathbf{P a})$ & $\begin{array}{c}\text { Tixotropia } \\
(\mathbf{P a} / \mathbf{s})\end{array}$ & $\begin{array}{c}\boldsymbol{\eta} \\
(\mathbf{m P a . s})\end{array}$ & $\boldsymbol{\lambda}(\boldsymbol{\mu m})$ & $\begin{array}{c}\text { Densidade a } \\
\text { verde (\% D.T.) }\end{array}$ \\
\hline 10 & 0,19 & 1158 & 4,00 & 4,20 & $37,05 \pm 0,34$ \\
\hline 15 & 0,43 & 2112 & 8,00 & 2,64 & $36,57 \pm 0,44$ \\
\hline 20 & 0,87 & 3511 & 16,00 & 1,87 & $35,26 \pm 0,27$ \\
\hline $\mathbf{2 5}$ & $\mathbf{3 , 6 5}$ & $\mathbf{5 9 2 5}$ & $\mathbf{4 8 , 0 0}$ & $\mathbf{1 , 4 0}$ & $\mathbf{3 6 , 7 9} \pm \mathbf{1 , 7 1}$ \\
\hline 30 & 5,93 & 45260 & 93,00 & 1,09 & $33,53 \pm 1,51$ \\
\hline
\end{tabular}

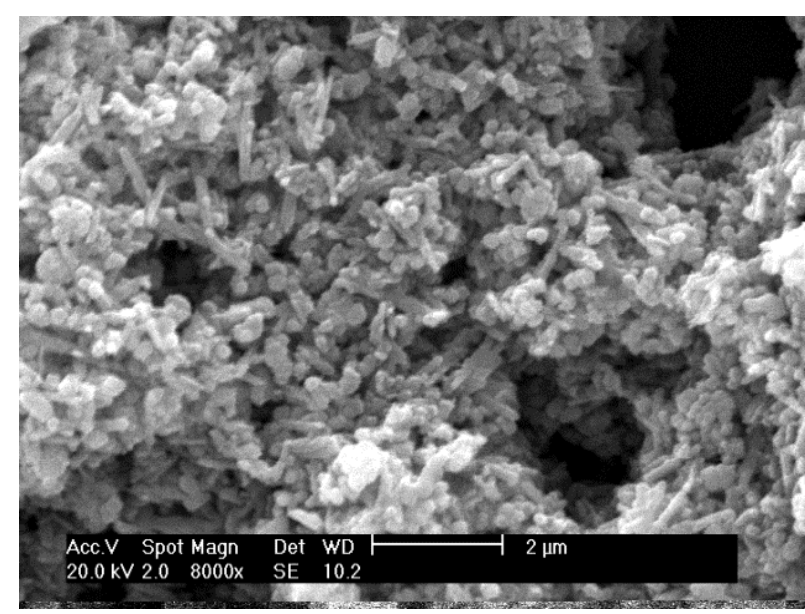

Figura 5: Micrografias das partículas de hematita processadas por colagem de barbotina.

\section{CONCLUSÕES}

$\mathrm{O}$ pH das suspensões exerceu influência no processo de aglomeração das partículas de hematita devido à redução do valor absoluto do potencial zeta. Ambos os polieletrólitos reduziram a viscosidade das suspensões, sendo o poliacrilato de sódio (PANa) o mais efetivo. As suspensões exibiram comportamento pseudoplástico. Quanto à quantidade de defloculante, a concentração escolhida de PANa foi de $2,0 \% \mathrm{~m} / \mathrm{m}$ devido à combinação das características de viscosidade e densidade dos corpos de prova a verde. Chegou-se a máxima concentração de sólidos de $30 \%$ vol. que correspondeu a uma consistência de pasta, inviável para o processamento por colagem. Com base nos resultados alcançados, a estabilização da suspensão aquosa de hematita se deu com $25 \% \mathrm{v} / \mathrm{v} ; 2 \% \mathrm{~m} / \mathrm{m}$ de poliacrilato de sódio e $\mathrm{pH}$ ajustado para 10 . As imagens de microscopia eletrônica de varredura revelaram a morfologia das partículas de hematita como bastonetes com tamanho inferior a $1 \mu \mathrm{m}$, com diâmetros em torno de $200 \mathrm{~nm}$. 


\section{BIBLIOGRAFIA}

[1] FIGUEROLA, A., DI CORATO, R., MANNA, L., et al., "From iron oxide nanoparticles towards advanced iron-based inorganic materials designed for biomedical applications", Pharmacological Research, v. 62 , p. $126-143,2010$.

[2] ALY, M. H., ISMAEL, I. S., BONDIOLI, F. "Synthesis of coloured ceramic pigments by using chromite and manganese ores mixtures", Cerâmica, v. 56, p. 156 - 161, 2010.

[3] ESCARDinO, A., MESTRE, S., BARBA, A., et al., "Kinetic study of black $\mathrm{Fe}_{2} \mathrm{O}_{3}-\mathrm{Cr}_{2} \mathrm{O}_{3}$ pigment synthesisiS I: influence of synthesis time and temperature", Journal of the American Ceramic Society, v. 86, p. $945-950,2003$.

[4] ESCARDINO, A., MESTRE, S., BARBA, A., et al., "Synthesis mechanism of an iron-chromium ceramic pigment", Journal of the American Ceramic Society, v. 83 , p. 29 - 32 , 2000.

[5] ZHENG, S., ZHANG, Q. "Surface-modification of fine red iron oxide pigment",China Particuology, v. 1, p. $176-180,2003$.

[6] NSIB, F., AYED, N., CHEVALIER, Y. "Dispersion of hematite suspensions with sodium polymethacrylate dispersants in alkaline medium", Colloids and Surfaces A: Physicochem. Eng. Aspects, v. 286 , p. $17-26,2006$.

[7] KANDORI, K., HORI, N., ISHIKAWA, T. "Preparation of mesoporous hematite particles by a forced hydrolysis reaction accompanying a peptide production reaction", Colloids and Surfaces A: Physicochem. Eng. Aspects, v. 290 , p. $280-287,2006$.

[8] LINDGREN, T., WANG, H., BEERMANN, N., et al., "Aqueous photoelectrochemistry of hematite nanorod array", Solar Energy Materials \& Solar Cells, v. 71, p. 231 - 243, 2002.

[9] GUO, P., WEI, Z., WANG, B., et al.,"Controlled synthesis, magnetic and sensing properties of hematite nanorods and microcapsules", Colloids and Surfaces A: Physicochem. Eng. Aspects, v. 380, p. 234 - 240, 2011.

[10] HU, Y., KLEIMANN-SHWARCSTEIN, A., FORMAN, A.J., et al., "Pt-doped a-Fe $\mathrm{O}_{3}$ thin films active for photoelectrochemical water splitting", Chemistry of Materials, v. 20, p. 3803 - 3805, 2008.

[11] VERA, P., GALLARDO, V., SALCEDO, J., DELGADO, A.V.,"Adsorption of a corticoid on colloidal hematite particles of different geometries", Journal of Colloid and Interface Science, v. 187, p. 429 - 434 , 1997.

[12] HOTZA, D. "Artigo revisão: Colagem de folhas cerâmicas", Cerâmica, v. 43, p. 283 - 284, 1997.

[13] LEWIS, J. A. "Colloidal processing of ceramics", Journal of the American Ceramic Society, v. 83 , p. $2341-2359$, (2000).

[14] SÁNCHEZ-HERENCIA, A. J. "Water Based Colloidal Processing of Ceramic Laminates", Key Engineering Materials, v. 333, p. 39 - 48, 2007.

[15] OllVEIRA, I. R., STUDART, A.R., PILEGGI, R.G., PANDOLFELli, V.C., Dispersão e Empacotamento de Partículas. São Paulo: Fazendo Arte, 2000.

[16] SALES, L. L. M., SOUZA, A.G., SOLEDADE, L.E.B., et al., "Influência do pH sobre a estabilidade de suspensões de alumina estabilizadas eletrostericamente", Química nova, v. 1, p. 70 - 74, 2007.

[17] DE NONI JR, A., GARCIA, D. E., HOTZA, D. "A modified model for the viscosity of ceramic suspensions", Ceramics International, v. 28, p. 731 - 735, 2002.

[18] GUEDES, C. D., PEREIRA, J.G, DE LENA, J.C., et al., "Coagulação/floculação de suspensões ricas em óxidos de ferro por sulfato de alumínio", Química Nova, v. 27, p. 715 - 719, 2004.

[19] RUFIER, C., REUFER, M., DIETSCH, H., et al., "Single step hybrid coating process to enhance the electrosteric stabilization of inorganic particles", Langmuir, v. 27, p. 6622 - 6627, 2011.

[20] ISRAELACHVILI, J. Intermolecular \& Surface Forces. 2. ed. London: Academic Press, 1991.

[21] REED, J. S. Principles of Ceramic Processing. New York: John Wiley \& Sons, 1995.

[22] ALMEIDA, K. A., MARTINS, L., CARDOSO, D. "Preparação e propriedades de zeólitas faujasitas contendo cátions amônio", Química Nova, v. 33, p. 1077 - 1081, 2010. 
SCIREA, R. F.; VIEIRA JUNIOR, L. E.; RODRIGUES NETO, J. B.; HOTZA, D. revista Matéria, v. 20, n. 1, pp. 185 - 192, 2015.

[23] BERGSTRÖM, K. Colloidal processing of ceramics, In: Handbook of Applied Surface and Colloid Chemistry.[s.1.]: John Wiley \& Sons, 2002. Cap. 9, p. 1065. 